\title{
Evaluation of the Psychological Status, Infertility-Associated Factors, and Erectile Function in Patients with Timely Ovulatory Intercourse Failure in China: Evidence from a Cross-Sectional Study
}

\author{
Jian Xiong, ${ }^{1}$ Zhonglin Cai, $^{1}$ Chengquan Ma, ${ }^{1}$ Bin Yang, ${ }^{1,2}$ Jianzhong Zhang $\left(\mathbb{D},{ }^{3}\right.$ \\ and Hongjun $\mathrm{Li}$ iD \\ ${ }^{1}$ Department of Urology, Peking Union Medical College Hospital, Peking Union Medical College, Chinese Academy of \\ Medical Sciences, Beijing, China \\ ${ }^{2}$ Department of Urology, Affiliated Hospital of the Qingdao University, Qingdao, China \\ ${ }^{3}$ Department of Urology, Beijing Friendship Hospital, Capital Medical University, China \\ Correspondence should be addressed to Jianzhong Zhang; 15895827758@163.com and Hongjun Li; lihongjun@pumch.cn
}

Received 1 May 2020; Revised 26 August 2020; Accepted 3 September 2020; Published 21 September 2020

Academic Editor: Andr Talvani

Copyright (c) 2020 Jian Xiong et al. This is an open access article distributed under the Creative Commons Attribution License, which permits unrestricted use, distribution, and reproduction in any medium, provided the original work is properly cited.

\begin{abstract}
Background and Objective. Knowledge on the clinical features of timely ovulatory intercourse failure (TOIF) is limited. The current study is aimed at investigating the psychological status, infertility-associated factors, and erectile function in males with TOIF. Material and Methods. Between June 2018 and December 2018, all qualified patients of infertile couples were continuously enrolled from the andrologic clinic of Peking Union Medical College Hospital. Semen analyses and demographic data were collected, and the International Index of Erectile Function 15 (IIEF-15) questionnaire was used to evaluate the erectile function in the participants with or without TOIF. In addition, the Mental Health Inventory- 5 and State-Trait Anxiety Inventory-Short Form questionnaires were used to evaluate the psychological status. Results. A total of 1128 patients were enrolled, and 264 of them $(23.40 \%)$ suffered from TOIF. TOIF was positively associated with smoking, drinking, hypertension, diabetes, anxiety, unknown cause of infertility, lower semen concentration, lower frequency of intercourse, and decreased erectile function. The total IIEF-15 scores, erectile function, orgasmic function, sexual desire, intercourse satisfaction, and overall satisfaction were significantly decreased in patients with TOIF. Conclusion. TOIF is associated with lower semen concentration, anxiety, and other comorbidities such as hypertension and diabetes. Clinicians are required to focus on these associated factors in addition to improve the erectile function.
\end{abstract}

\section{Introduction}

Timely ovulatory intercourse failure (TOIF), which occurs when the males failed to penetrate their wives' vagina during the ovulatory time, may affect physical and psychosocial health and may have a significant influence on the quality of life of the patients and their partners [1]. TOIF is not a rare disorder in males of infertile couples, and epidemiological data have shown a high prevalence of TOIF in males of infertile couples in China. In 2018, Yang et al. first put forward the concept of TOIF and reported that $26.2 \%$ of the males using timely ovulatory intercourse suffered from TOIF [1]. However, although the prevalence of TOIF is high, there were only few studies that have focused on this issue and there were no clear definitions. Based on the previous study by Yang et al., a patient diagnosed with TOIF should fulfill the following criteria: (1) were aware of their partners' ovulatory time, (2) had tried sexual intercourse during their partners' ovulatory time, and (3) experienced at least three failed attempts. In the current study, we have utilized the same criteria.

Knowledge about the associated factors of TOIF is limited. A previous study has reported an association between 
TOIF and erectile dysfunction (ED) by using the 5-item version of the International Index of Erectile Function (IIEF-5) questionnaire. The prevalence of TOIF in males with ED $(23.3 \%)$ was significantly higher than that in those without ED (8.6\%). Notably, TOIF did not solely result from ED considering its nature that is firmly associated with the partners' ovulatory time. Under the traditional Chinese culture, men take the responsibility to add a generation to carry on the family line. As infertility goes on, failures of timely ovulatory sexual intercourse exacerbate the psychological distress substantially [2-4]. The prevalence of psychological disorders is high in infertile males of Chinese couples. A recent study reported that the prevalence of depression, anxiety, and a combination of both psychological symptoms was $20.8 \%$, $7.8 \%$, and $15.4 \%$, respectively, in 771 infertile Chinese males [5]. Psychological stress from the traditional beliefs in China can be an important cause of TOIF. In addition, other infertility-associated factors such as duration of infertility, cause for infertility, and semen analysis results should not be ignored since these factors are directly associated with the psychological stress of these patients $[6,7]$.

The International Index of Erectile Function 15 (IIEF-15) questionnaire contains five parts: erectile function (IIEF-EF), orgasmic function, sexual desire, intercourse satisfaction, and overall satisfaction domain [8]. In addition to the erectile function, the orgasmic function, the sexual desire, and the patients' subjective satisfaction of their sexual intercourse can be recorded. Considering the prevalence of the psychological distress in males of infertile couples, the subjective feelings including the satisfaction and sexual desire and orgasmic function of the patients should be focused on.

In the current study, the IIEF-15 questionnaire was used to investigate the sexual function in males with or without TOIF so as to evaluate the subjective attitudes towards their sexual intercourse. In addition, data concerning the clinical features of the infertile patients, the psychological status, and the semen analysis results were collected to explore the potential associated factors of TOIF.

\section{Materials and Methods}

2.1. Study Design and Participants. This cross-sectional and clinic-based study focused on the male partners of the infertile couples who failed to conceive after 1 year or more of regular unprotected sexual intercourse. The participating patients were continuously enrolled from the urologic clinic of Peking Union Medical College Hospital from June 1, 2018, through December 1, 2018. The inclusion criteria were (1) men living day to day with their wives or common-law partners and planning to have a baby, (2) females failing to achieve a clinical pregnancy after engaging in regular unprotected sexual intercourse for at least 1 year, (3) men attending the andrologic clinics for infertility with at least two semen samples analyzed, and (4) men who were aware of the ovulatory time of their female partners and using timely ovulatory intercourse. The patients were excluded when they were (1) males with severe cardiovascular diseases, mental illness such as dementia and delirium, or visible genital malformation and (2) men whose wives had abnormality of the reproduc- tive system based on gynecologic examination. The males of infertile couples would be diagnosed with TOIF if they (1) were aware of their partners' ovulatory time, (2) had tried sexual intercourse during their partners' ovulatory time, and (3) experienced at least three failed attempts. The study was completely anonymous and voluntary, and informed consent forms were obtained from all the patients. This study was approved by the Research Ethics Committee in Peking Union Medical College Hospital (ethical number: JS-1176).

2.2. Questionnaire Survey and Semen Analyses. A selfdesigned questionnaire based on the previous study [1] was used to collect the basic information of the enrolled patients including age, height, weight, residence (urban or rural), monthly income, education level, occupation (office work, labor work, or others), smoking, alcohol drinking, marital status (primary or secondary), prevalence of coexisting diseases (hypertension, diabetes, varicocele, and chronic prostatitis), type of infertility (primary or secondary), duration of infertility, cause of infertility (known or unknown), frequency of sexual intercourse, and prevalence of TOIF.

The erectile function was evaluated by IIEF-15, which includes five domains: erectile function (EF domain; 1-30), orgasmic function (OF domain; 0-10), sexual desire (SD domain; 2-10), intercourse satisfaction (IS domain; 0-15), and overall satisfaction (OS domain; 2-10). The severity of ED was defined based on the EF domain value: severe ED (EF domain <11), moderate ED (EF domain 11-16), mild to moderate $\mathrm{ED}$ (EF domain 17-21), mild ED (EF domain 22-25), and no ED (EF domain 26-30). In addition, the prevalence of depression and anxiety was analyzed by two structured, self-report questionnaires, the Mental Health Inventory-5 (MHI-5) and the Six-Item State-Trait Anxiety Inventory-Short Form (STAI-6), respectively. Depression was primarily considered when the total score of MHI-5 was 52 or lower, and when the total score of STAI- 6 equaled to 45 or higher, the patient was diagnosed with anxiety.

All the participants provided at least two semen samples, and the mean values were recorded. Semen analyses were carried out strictly according to the WHO Laboratory Manual for the Examination and Processing of Human Semen (fifth edition, 2010).

2.3. Statistical Analysis. Patients were divided into two groups based on whether they had ever suffered from TOIF in the last 3 months. Categorical and continuous data were presented as the frequency and mean \pm standard deviation, respectively. The chi-squared test was used to compare categorical data between the two groups. The two-tailed Student's $t$-test was used for normally distributed continuous data, and Mann-Whitney's $U$ test was used for nonnormally distributed data. Potential factors associated with the occurrence of TOIF were identified by multivariable binary logistic regression analyses. The specific association was evaluated by the adjusted odds ratios (ORs) with their corresponding 95\% confidence intervals (CIs). The results were considered significant when two-tailed $p$ values $<0.05$. The statistical analysis was performed using Stata version 12 (StataCorp LP, College Station, TX). 


\section{Results}

3.1. Basic Characteristics of the Enrolled Patients. A total of 1128 males who were aware of their female partners' ovulatory time were enrolled, and 264 of them (23.40\%) suffered from TOIF. The basic information is listed in Table 1 . The mean age was significantly higher in patients with TOIF $(p<0.001)$. Based on the results of the chi-squared test, occurrence of TOIF in males with office work $(p=0.011)$, higher monthly income $(p<0.001)$, smoking $(p=0.004)$, and drinking habits $(p<0.001)$ was significantly higher. In terms of the unique variables of the infertile couples, patients with moderate or worse couple's relationship $(p=0.001)$, lower frequency of sexual intercourse $(p<0.001)$, unknown cause of infertility $(p=0.001)$, lower sperm concentration $(p<0.001)$, and lower progressive motility $(p<0.001)$ were more likely to have TOIF. In terms of the psychological factors, TOIF was associated with anxiety $(p<0.001)$ but not with depression $(p=0.391)$. Besides, the prevalence of hypertension $(p<0.001)$, diabetes $(p=0.024)$, and chronic prostatitis $(p=0.001)$ increased in patients with TOIF.

3.2. Factors Associated with TOIF. Possible associated factors for TOIF were further identified by multivariate analysis. The occurrence of TOIF was positively associated with drinking habits (OR: $1.62,95 \% \mathrm{CI}: 1.14$ to $2.29, p=0.007$ ), unknown cause of infertility (OR: $1.65,95 \%$ CI: 1.11 to $2.44, p=0.013$ ), hypertension (OR: $2.35,95 \% \mathrm{CI}: 1.14$ to $4.86, p=0.021$ ), diabetes (OR: $1.53,95 \% \mathrm{CI}: 1.00$ to $2.02, p=0.050$ ), anxiety (OR: $4.51,95 \%$ CI: 2.44 to $8.33, p<0.001)$, lower frequency of sexual intercourse (OR: $1.82,95 \% \mathrm{CI}: 1.31$ to $2.53, p<0.001$ ), lower sperm concentration (OR: $1.51,95 \%$ CI: 1.03 to 2.21 , $p=0.035$ ), and erectile dysfunction (OR: 3.89, 95\% CI: 2.82 to $5.35, p<0.001)$. The detailed information is listed in Table 2.

3.3. Erectile Function of the Patients with or without TOIF. The IIEF-15 questionnaire was used to evaluate the erectile function of patients with or without TOIF. Results indicated that the mean EF domain, OF domain, SD domain, IS domain, OS domain, and total score were 22.62 $\pm 4.96,6.97$ $\pm 1.70,6.00 \pm 1.67,10.22 \pm 2.38,6.67 \pm 2.57$, and $52.48 \pm$ 10.71 , respectively, and all of these values were significantly lower than the corresponding indicators in patients without TOIF $(p<0.001)$ (Figure 1). Not only the erectile function but also the subjective feelings including the orgasmic function, sexual desire, intercourse satisfaction, and overall satisfaction were significantly decreased.

Patients were further grouped into no ED, mild ED, mild to moderate $\mathrm{ED}$, moderate $\mathrm{ED}$, and severe $\mathrm{ED}$ to investigate the association between severity of ED and TOIF occurrence. In patients with TOIF, 84 (31.82\%) did not have ED, 84 $(31.82 \%)$ had mild ED, 65 (24.62\%) had mild to moderate ED, 25 (9.47\%) had moderate ED, and 6 (2.27\%) had severe ED. In patients without TOIF, $595(68.87 \%)$ did not have ED, 196 (22.67\%) had mild ED, 57 (6.60\%) had mild to moderate ED, 14 (1.63\%) had moderate ED, and $2(0.23 \%)$ had severe ED. Results demonstrated that the prevalence of TOIF was positively associated with the severity of ED (Figure 2).

\section{Discussion}

Male infertility is a complex problem and affects approximately $7 \%$ of the males in modern society. Failure to penetrate the vagina during the sexual partner's ovulatory time can be one common cause for infertility. The concept of TOIF has been put forward by Yang et al. in 2018 [1]. The study which enrolled 4299 patients from 29 urologic clinics in different regions of China reported that $26.2 \%$ of the males with timely ovulatory sexual intercourse have TOIF. According to the existing data, TOIF is common and has affected $23.4 \%$ of the males of infertile couples. The occurrence is similar to the results of the previous study. Considering the traditional culture of China, males were uppermost in a marital relation and took the responsibility to add a generation to the family tree. The psychological burden of the Chinese young male can be high and subsequently induces a high prevalence of TOIF. Currently, epidemiological data of TOIF in other areas is limited and requires further evaluation.

According to a previous published meta-analysis which investigated the association between lifestyle and sexual function, patients with cigarette smoking and alcohol drinking have 1.41 times and 1.19 times risk of ED, respectively [9]. In the current study, multivariate analyses indicated that smoking and alcohol drinking are also independent associated factors for TOIF. Lifestyle education is important during the treatment of TOIF. Large amounts of clinical studies have demonstrated the association between chronic diseases and ED [10-12]. Patients with cardiovascular diseases or diabetes are more likely to have ED because these conditions share similar associated factors and potential mechanisms [13, 14]. In this cross-sectional study, patients suffering from hypertension and diabetes were more likely to have TOIF. Strengthening the breadth and depth of chronic diseases (such as hypertension and diabetes) management may improve the prognosis of TOIF.

Psychological stress plays an important role in reproductive health and can even affect artificial reproductive technology success [15]. The prevalence of psychological disorders is high in Chinese males of infertile couples, and several studies have reported the negative impact of psychological factors on infertile couples. In 2015, Yang et al. demonstrated that the prevalence of depression and anxiety was $20.8 \%$ and $7.8 \%$, respectively, in 771 infertile Chinese males [5]. Compared with patients without psychological disorders, patients with anxiety are more likely to have ED. In the current population, the prevalence of anxiety and depression was similar to this previous study. Notably, the prevalence of anxiety was significantly higher in patients with TOIF. Inability to penetrate the partner's vagina and ejaculation has a direct impact on infertility. When the infertile man experiences TOIF, psychological distress is elevated for both himself and his sexual partner, leading to increased susceptibility to anxiety. In addition to the erectile function and semen analysis results, physicians should strengthen the psychological counseling and humanistic care of the patients.

In terms of the factors associated with infertility, the results indicated that TOIF was positively associated with unknown cause of infertility and lower sperm concentration. 
TABLE 1: Basic characteristics of the patients with or without TOIF.

\begin{tabular}{|c|c|c|c|}
\hline \multirow{2}{*}{ Parameters } & \multicolumn{2}{|c|}{ Subjects } & \multirow{2}{*}{$p$ value } \\
\hline & TOIF $(n=264)$ & Non-TOIF $(n=864)$ & \\
\hline Age $(y)$, mean $\pm S D$ & $34.39 \pm 6.19$ & $32.18 \pm 5.25$ & $<0.001$ \\
\hline $\mathrm{BMI}\left(\mathrm{kg} / \mathrm{m}^{2}\right)$, mean $\pm \mathrm{SD}$ & $24.71 \pm 3.13$ & $24.81 \pm 3.18$ & 0.595 \\
\hline Residence & & & 0.118 \\
\hline Rural & $32(12.12)$ & $141(16.32)$ & \\
\hline Urban & $232(87.88)$ & $723(83.68)$ & \\
\hline Education level, $n(\%)$ & & & 0.996 \\
\hline No higher than junior high school & $24(9.09)$ & $77(8.91)$ & \\
\hline High school & $47(17.80)$ & $154(17.82)$ & \\
\hline University and above & $193(73.11)$ & $633(73.27)$ & \\
\hline Monthly income (RMB), $n(\%)$ & & & $<0.001$ \\
\hline$<5000$ & $78(29.55)$ & $403(46.64)$ & \\
\hline $5001-10000$ & $116(43.94)$ & $310(35.88)$ & \\
\hline $10001-20000$ & $46(17.42)$ & $103(11.92)$ & \\
\hline$>20000$ & $24(9.09)$ & $48(5.56)$ & \\
\hline Occupation, $n(\%)$ & & & 0.011 \\
\hline Office work & $141(53.41)$ & $398(46.07)$ & \\
\hline Manual labor work & $71(26.89)$ & $216(25.00)$ & \\
\hline Other & $52(19.70)$ & $250(28.93)$ & \\
\hline Smoking, $n(\%)$ & & & 0.004 \\
\hline Yes & $106(40.15)$ & $267(30.90)$ & \\
\hline No & $158(59.85)$ & $597(69.10)$ & \\
\hline Alcohol consumption, $n(\%)$ & & & $<0.001$ \\
\hline Yes & $112(42.42)$ & $239(27.66)$ & \\
\hline No & $152(57.58)$ & $625(72.34)$ & \\
\hline Couple's relationship, $n(\%)$ & & & 0.001 \\
\hline Good & $238(90.15)$ & $828(95.83)$ & \\
\hline Moderate or worse & $26(9.85)$ & $36(4.17)$ & \\
\hline Marital status, $n(\%)$ & & & 0.292 \\
\hline Primary & $250(94.70)$ & $827(95.72)$ & \\
\hline Secondary & $14(5.30)$ & $37(4.28)$ & \\
\hline Frequency of intercourse ( $n /$ month), $n(\%)$ & & & $<0.001$ \\
\hline$<5$ & $126(47.73)$ & $235(27.20)$ & \\
\hline $5-8$ & $113(42.80)$ & $416(48.15)$ & \\
\hline$>9$ & $25(9.47)$ & $213(24.65)$ & \\
\hline \multicolumn{4}{|l|}{ Medical history } \\
\hline Type of infertility, $n(\%)$ & & & 0.182 \\
\hline Primary & $182(68.94)$ & $633(73.26)$ & \\
\hline Secondary & $82(31.06)$ & $231(26.74)$ & \\
\hline Duration of infertility, $n(\%)$ & & & 0.101 \\
\hline$<2$ & $70(26.52)$ & $205(23.73)$ & \\
\hline $2-4$ & $112(42.42)$ & $431(49.88)$ & \\
\hline$>4$ & $82(31.06)$ & $228(26.39)$ & \\
\hline Cause of infertility, $n(\%)$ & & & 0.001 \\
\hline Known & $192(72.73)$ & $709(82.06)$ & \\
\hline Unknown & $72(27.27)$ & $155(17.94)$ & \\
\hline Hypertension, $n(\%)$ & & & $<0.001$ \\
\hline Yes & $24(9.09)$ & $22(2.55)$ & \\
\hline No & $240(90.91)$ & $842(97.45)$ & \\
\hline
\end{tabular}


TABle 1: Continued.

\begin{tabular}{|c|c|c|c|}
\hline \multirow{2}{*}{ Parameters } & \multicolumn{2}{|c|}{ Subjects } & \multirow{2}{*}{$p$ value } \\
\hline & TOIF $(n=264)$ & Non-TOIF $(n=864)$ & \\
\hline Diabetes, $n(\%)$ & & & 0.024 \\
\hline Yes & $9(3.41)$ & $10(1.16)$ & \\
\hline No & $255(96.59)$ & $854(98.84)$ & \\
\hline Chronic prostatitis, $n(\%)$ & & & 0.001 \\
\hline Yes & $47(17.80)$ & $90(10.42)$ & \\
\hline No & $217(82.20)$ & $774(89.58)$ & \\
\hline Varicocele, $n(\%)$ & & & 0.060 \\
\hline Yes & $72(27.27)$ & $291(33.68)$ & \\
\hline No & $192(72.73)$ & $573(66.32)$ & \\
\hline Depression, $n(\%)$ & & & 0.391 \\
\hline Yes & $38(14.39)$ & $145(16.78)$ & \\
\hline No & $226(85.61)$ & $719(83.22)$ & \\
\hline Anxiety, $n(\%)$ & & & $<0.001$ \\
\hline Yes & $38(14.39)$ & $34(3.94)$ & \\
\hline No & $226(85.61)$ & $830(96.06)$ & \\
\hline \multicolumn{4}{|l|}{ Semen quality, $n(\%)$} \\
\hline Sperm concentration $\left(10^{6} / \mathrm{mL}\right), n(\%)$ & & & $<0.001$ \\
\hline$<5$ & $38(14.39)$ & $32(3.70)$ & \\
\hline $5-15$ & $64(24.24)$ & $247(28.59)$ & \\
\hline$>15$ & $162(61.37)$ & $585(67.71)$ & \\
\hline Progressive motility (\%), $n(\%)$ & & & $<0.001$ \\
\hline$<5$ & $17(6.44)$ & $14(1.62)$ & \\
\hline $5-20$ & $37(14.01)$ & $67(7.76)$ & \\
\hline $20-32$ & $102(38.64)$ & $381(44.10)$ & \\
\hline$>32$ & $108(40.91)$ & $402(46.52)$ & \\
\hline
\end{tabular}

TABLE 2: Multivariate analysis of the association between study variables and TOIF occurrence.

\begin{tabular}{lccc}
\hline Parameters & Adjusted OR & $95 \%$ CI & $p$ value \\
\hline Age $>32$ years & 1.27 & 0.90 to 1.79 & 0.177 \\
Smoking & 1.42 & 1.00 to 2.02 & 1.14 to 2.29 \\
Drinking & 1.62 & 0.92 to 2.04 & 0.052 \\
Monthly income $>10000 \mathrm{RMB}$ & 1.37 & 0.86 to 1.64 & 0.007 \\
Office work & 1.19 & 0.67 to 1.40 \\
Secondary infertility & 0.97 & 1.11 to 2.44 \\
Unknown cause of infertility & 1.65 & 1.14 to 4.86 & 0.124 \\
Hypertension & 2.35 & 1.00 to 2.02 & 0.890 \\
Diabetes & 1.53 & 0.892 .14 \\
Chronic prostatitis & 1.38 & 0.83 to 1.32 \\
Varicocele & 1.05 & 2.44 to 8.33 \\
Anxiety & 4.51 & 0.48 to 1.12 & 0.013 \\
Depression & 0.73 & 1.31 to 2.53 \\
Frequency of intercourse $<5 / \mathrm{month}$ & 1.82 & 1.03 to 2.21 \\
Sperm concentration $<15 * 10^{6} / \mathrm{mL}$ & 1.51 & 0.82 to 1.68 & 0.050 \\
Progressive motility $<32 \%$ & 1.17 & 2.82 to 5.35 \\
IIEF-EF $<26$ & 3.89 & 0.152 \\
\hline
\end{tabular}



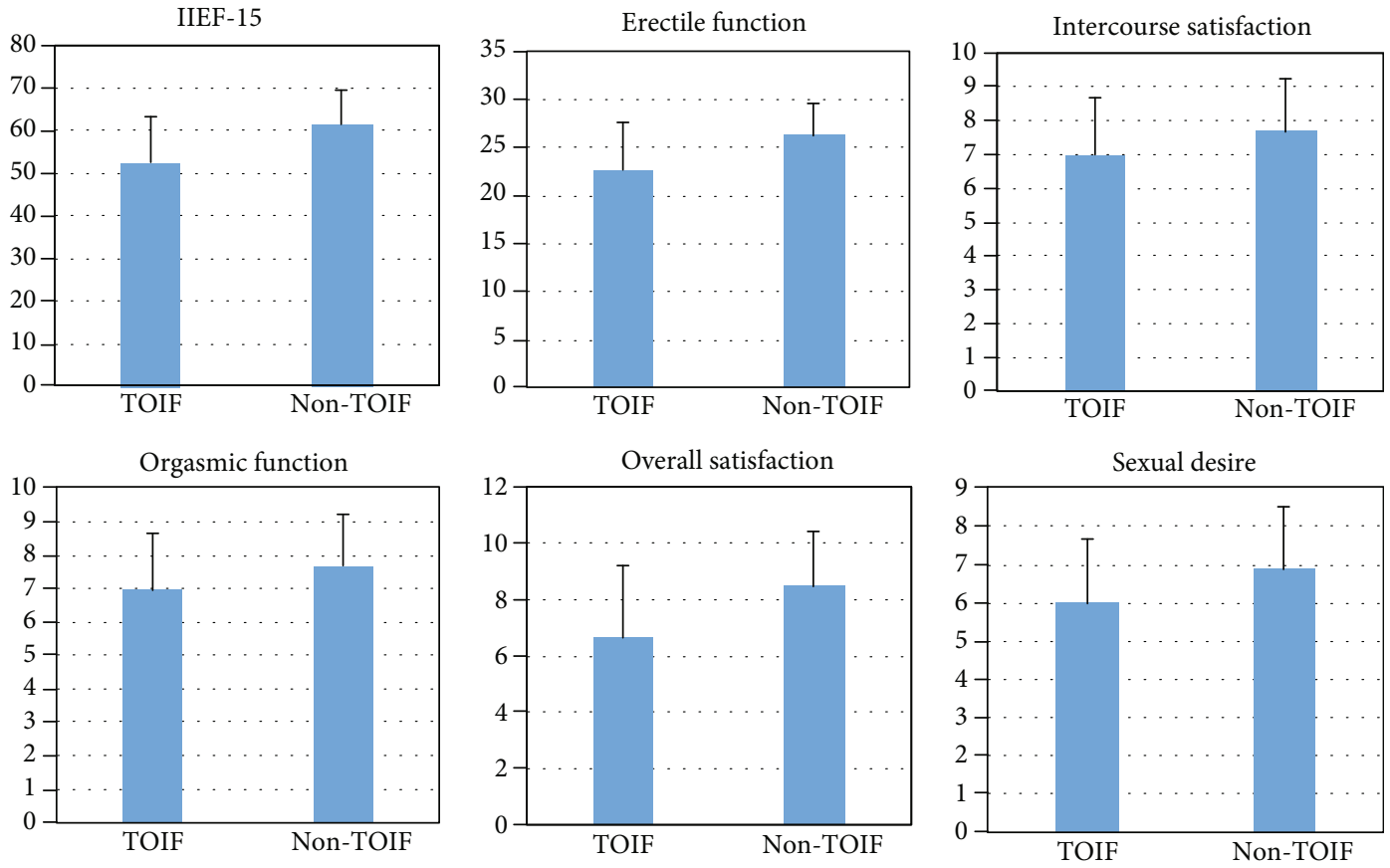

Figure 1: Erectile function of the patients with or without TOIF. ${ }^{*} p<0.001$ in all the groups.

\begin{tabular}{lccccc}
\hline & \multirow{2}{*}{ No ED } & Mild ED & Mild to moderate ED & Moderate & Severe \\
& & & & ED & ED \\
\hline TOIF & 84 & 84 & 65 & 25 & 6 \\
Non-TOIF & 595 & 196 & 57 & 14 & 2 \\
\hline
\end{tabular}

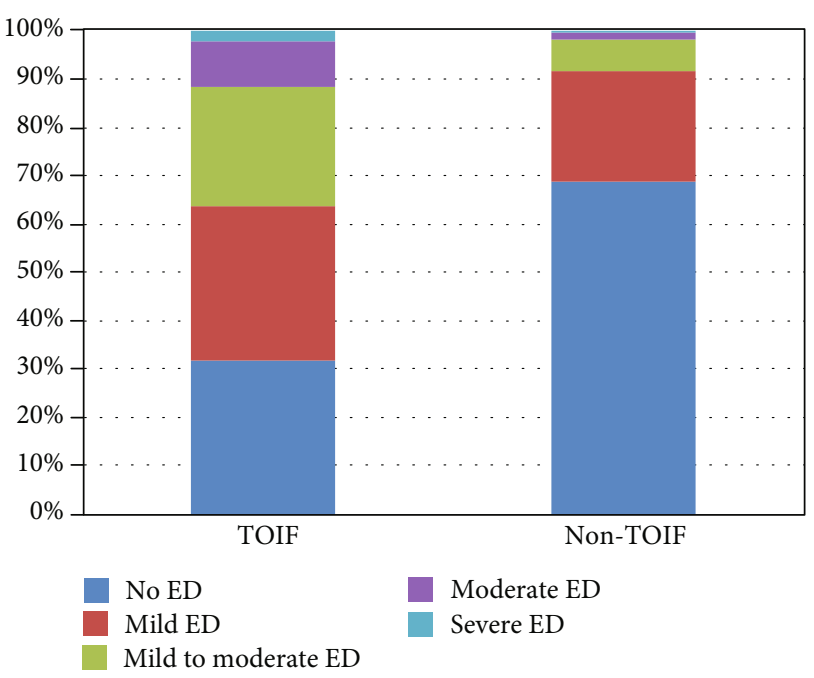

Figure 2: Association between the severity of ED and TOIF. ${ }^{*} p<$ 0.001 .

The sperm concentration can directly reflect the severity of male infertility. The modern assisted reproductive technique (ART) allows patients with severe oligozoospermia to have their own child by using just tiny amounts of sperm [16, 17]. However, the management of patients with azoospermia is still challenging and the reproductive outcomes are usually unsatisfied. In addition, the fear of unknown causes of infertility can also increase the psychological stress of these patients. These factors may affect the erectile function and subsequently result in TOIF. In addition, these factors of infertility are firmly associated with testicular dysgenesis syndrome (TDS). TDS is also a risk factor of ED or worsened ED and may subsequently result in TOIF.

ED is common in males with infertility. The prevalence of ED has been reported by several studies, ranging from $18 \%$ to $57 \%[18,19]$. The association between ED and TOIF has also been identified. Similar to the results of Dr. Yang et al. [1], ED is an independent associated factor for TOIF in the current study. To further investigate the subjective satisfaction of the patients with TOIF, the IIEF-15 questionnaire was used. The results indicated that the total IIEF-5 scores, erectile function, orgasmic function, sexual desire, intercourse satisfaction, and overall satisfaction were significantly decreased in patients with TOIF. Not only the erectile function but also the subjective feelings of the patients should be focused on. Notably, severe ED just occupied a small proportion in patients with male infertility, ranging from 2.4 to $2.9 \%[20,21]$. In the current population, severe ED accounts for $2.27 \%$ of the patients with TOIF. Most of these patients suffered from mild or mild to moderate ED. The severity of ED is positively associated with TOIF occurrence. While clinicians should focus on the overall satisfaction of the patients, treatment of ED is also very important, especially in those with severe ED.

There were several strengths of the current study. First, a relatively large number of patients were enrolled which enabled further multivariate analyses. Second, semen analyses and psychological status were analyzed in all the participants. Despite the aforementioned advantages, sexual hormones were not detected and this was a limitation. Concerning the important role of hormones, including sexual hormones, thyroxine, and melatonin, in erectile function, it is necessary for future studies researching on this field. 


\section{Conclusions}

TOIF is associated with lower semen concentration, anxiety, and other comorbidities including hypertension and diabetes. Clinicians are required to focus on these associated factors in addition to improve the erectile function of the males of infertile couples.

\section{Abbreviations}

TOIF: Timely ovulatory intercourse failure

ED: $\quad$ Erectile dysfunction

MHI-5: Mental Health Inventory-5

STAO-6: Six-Item State-Trait Anxiety Inventory-Short Form

IIEF-15: International Index of Erectile Function 15.

\section{Data Availability}

The data supporting the conclusions of this article is included within the article.

\section{Ethical Approval}

The current study was approved by the ethical committee of Peking Union Medical College Hospital (ethical number: JS-1176).

\section{Consent}

All participants signed an informed consent.

\section{Conflicts of Interest}

The authors declare that they have no competing interests.

\section{Authors' Contributions}

The contributions of the authors involved in this study are as follows: HJ Li: project development and manuscript writing; JZ Zhang: data collection and manuscript writing; J Xiong: data analysis, data collection, manuscript writing, and revision; ZL Cai: data analysis and data collection; CQ Ma: data analysis and data collection; and B Yang: data analysis and data collection.

\section{Acknowledgments}

This work is supported by the grant from the National Natural Science Foundation of China (Grant Nos. 81671448 and 81871152) and CAMS Innovation Fund for Medical Sciences (Grant No. 2018-I2M-1-004).

\section{References}

[1] B. Yang, P. Xu, Y. Shi et al., "Erectile dysfunction and associated risk factors in Chinese males of infertile couples," The Journal of Sexual Medicine, vol. 15, no. 5, pp. 671-677, 2018.

[2] T. Y. Lee and G. H. Sun, "Psychosocial response of Chinese infertile husbands and wives," Archives of Andrology, vol. 45, no. 3, pp. 143-148, 2000.
[3] R. Cusatis, N. Fergestrom, A. Cooper et al., "Too much time? Time use and fertility-specific quality of life among men and women seeking specialty care for infertility," BMC Psychology, vol. 7, no. 1, p. 45, 2019.

[4] R. Omani-Samani, A. Ghaheri, B. Navid, M. Sepidarkish, and S. Maroufizadeh, "Prevalence of generalized anxiety disorder and its related factors among infertile patients in Iran: a cross-sectional study," Health and Quality of Life Outcomes, vol. 16, no. 1, p. 129, 2018.

[5] B. Yang, J. Zhang, Y. Qi, P. Wang, R. Jiang, and H. Li, “Assessment on occurrences of depression and anxiety and associated risk factors in the infertile Chinese men," American Journal of Men's Health, vol. 11, no. 3, pp. 767-774, 2017.

[6] A. Wdowiak, A. Bień, G. Iwanowicz-Palus, M. Makara-Studzińska, and I. Bojar, "Impact of emotional disorders on semen quality in men treated for infertility," Neuro Endocrinology Letters, vol. 38, no. 1, pp. 50-58, 2017.

[7] E. Vellani, A. Colasante, L. Mamazza, M. G. Minasi, E. Greco, and A. Bevilacqua, "Association of state and trait anxiety to semen quality of in vitro fertilization patients: a controlled study," Fertility and Sterility, vol. 99, no. 6, pp. 1565-1572.e2, 2013.

[8] R. C. Rosen, A. Riley, G. Wagner, I. H. Osterloh, J. Kirkpatrick, and A. Mishra, "The international index of erectile function (IIEF): a multidimensional scale for assessment of erectile dysfunction," Urology, vol. 49, no. 6, pp. 822-830, 1997.

[9] M. S. Allen and E. E. Walter, "Health-related lifestyle factors and sexual dysfunction: a meta-analysis of population-based research," The Journal of Sexual Medicine, vol. 15, no. 4, pp. 458-475, 2018.

[10] L. Luo, S. Zhao, J. Wang et al., "Association between chronic obstructive pulmonary disease and risk of erectile dysfunction: a systematic review and meta-analysis," International Journal of Impotence Research, vol. 32, no. 2, pp. 159-166, 2020.

[11] X. Y. Wang, W. Huang, and Y. Zhang, "Relation between hypertension and erectile dysfunction: a meta-analysisof cross-section studies," International Journal of Impotence Research, vol. 30, no. 3, pp. 141-146, 2018.

[12] Y. Kouidrat, D. Pizzol, T. Cosco et al., "High prevalence of erectile dysfunction in diabetes: a systematic review and meta-analysis of 145 studies," Diabetic Medicine, vol. 34, no. 9, pp. 1185-1192, 2017.

[13] X. Zhang, B. Yang, N. Li, and H. Li, "Prevalence and risk factors for erectile dysfunction in Chinese adult males," The Journal of Sexual Medicine, vol. 14, no. 10, pp. 1201-1208, 2017.

[14] Z. Cai, J. Zhang, and H. Li, “Two birds with one stone: regular use of PDE5 inhibitors for treating male patients with erectile dysfunction and cardiovascular diseases," Cardiovascular Drugs and Therapy, vol. 33, no. 1, pp. 119-128, 2019.

[15] F. J. Zhou, Y. N. Cai, and Y. Z. Dong, "Stress increases the risk of pregnancy failure in couples undergoing IVF," Stress, vol. 22, no. 4, pp. 414-420, 2019.

[16] The Practice Committee of the American Society for Reproductive Medicine, "Management of nonobstructive azoospermia: a committee opinion," Fertility and Sterility, vol. 110, no. 7, pp. 1239-1245, 2018.

[17] R. I. McLachlan, "Approach to the patient with oligozoospermia," The Journal of Clinical Endocrinology and Metabolism, vol. 98, no. 3, pp. 873-880, 2013.

[18] F. Lotti, G. Corona, G. Rastrelli, G. Forti, E. A. Jannini, and M. Maggi, "Clinical correlates of erectile dysfunction and 
premature ejaculation in men with couple infertility," The Journal of Sexual Medicine, vol. 9, no. 10, pp. 2698-2707, 2012.

[19] J. Gao, X. Zhang, P. Su et al., "Relationship between sexual dysfunction and psychological burden in men with infertility: a large observational study in China," The Journal of Sexual Medicine, vol. 10, no. 8, pp. 1935-1942, 2013.

[20] S. H. Song, D. S. Kim, T. K. Yoon, J. Y. Hong, and S. H. Shim, "Sexual function and stress level of male partners of infertile couples during the fertile period," BJU International, vol. 117, no. 1, pp. 173-176, 2016.

[21] A. Jungwirth, A. Giwercman, H. Tournaye et al., "European Association of Urology guidelines on male infertility: the 2012 update," European Urology, vol. 62, no. 2, pp. 324-332, 2012. 